\title{
GENERATING FUNCTIONS FOR FORMAL POWER SERIES IN NONCOMMUTING VARIABLES ${ }^{1}$
}

\section{K. GOLDBERG}

In [1] we stated that knowledge of the coefficients in the formal power series for $\log e^{x} e^{y}$ with $x y \neq y x$, could be translated into knowledge of the coefficients in $\log e^{f(x)} e^{o(y)}$ for any power series $f(x)$ and $g(y)$ with $f(0)=g(0)=0$. In the following we prove a generalization of this statement, valid for any formal power series in any number of noncommuting variables.

Let $A\left(x_{1}, \cdots, x_{n}\right)$ be an arbitrary formal power series in the noncommuting variables $x_{1}, \cdots, x_{n}$. It can be thought of as an element of the free associative ring generated by $x_{1}, \cdots, x_{n}$ over an arbitrary ring which contains its coefficients. For each positive integer $m$ let $V_{n m}$ be the set of ordered $m$-tuples $\left(s_{1}, \cdots, s_{m}\right)$ with each $s_{i}$ a positive integer no greater than $n$ and no two consecutive $s_{i}$ equal. Let $a_{s_{1}} \ldots s_{m}\left(i_{1}, \cdots, i_{m}\right)$, with each $i_{j}$ a positive integer, and $\left(s_{1}, \cdots, s_{m}\right)$ in $V_{n m}$, be the coefficient of $x_{s_{1}}^{i_{1}} \cdots x_{s_{m}}^{i_{m}}$ in $A\left(x_{1}, \cdots, x_{n}\right)$. Thus, if $a_{0}$ is the constant term, we can write $A\left(x_{1}, \cdots, x_{n}\right)$ uniquely as

$$
\begin{aligned}
& A\left(x_{1}, \cdots, x_{n}\right) \\
& \quad=a_{0}+\sum_{m=1}^{\infty} \sum_{\left(s_{i}\right) \in V_{n m}} \sum_{j=1}^{m} \sum_{i_{j}=1}^{\infty} a_{s_{1}} \ldots s_{m}\left(i_{1}, \cdots, i_{m}\right) x_{s_{1}}^{i_{1}} \cdots x_{s_{m}}^{i_{m}} .
\end{aligned}
$$

Now, for each positive integer $m$ and each $\left(s_{1}, \cdots, s_{m}\right)$ in $V_{n m}$ we define a correspondence $T_{s_{1}} \ldots s_{m}$ between $A\left(x_{1}, \cdots, x_{n}\right)$ and a generating function in commuting variables $z_{1}, \cdots, z_{m}$ as follows:

$$
T_{s_{1} \ldots s_{m}}: A\left(x_{1}, \cdots, x_{n}\right) \rightarrow \sum_{j=1}^{m} \sum_{i_{j}=1}^{\infty} a_{s_{1}} \cdots s_{m}\left(i_{1}, \cdots, i_{m}\right) z_{1}^{i_{1}} \cdots z_{m}^{i_{m}} .
$$

This correspondence can be thought of as a linear transformation which takes $a x_{s_{1}}^{i_{1}} \cdots x_{s_{m}}^{i_{m}}$ into $a z_{1}^{i_{1}} \cdots z_{m}^{i_{m}}$ for all positive $i_{1}, \cdots, i_{m}$, and takes all the other monomials into 0 .

We shall prove that this correspondence obeys the following rule.

TheOREM. Let $f_{1}(x), \cdots, f_{n}(x)$ be any formal power series with constant terms equal to 0 . If $A\left(x_{1}, \cdots, x_{n}\right)$ is a formal power series in

Received by the editors April 11, 1959 and, in revised form, November 13, 1959.

1 The preparation of this paper was supported (in part) by the Office of Naval Research. 
noncommuting variables $x_{1}, \cdots, x_{n}$ and

$$
T_{s_{1} \cdots s_{m}}: A\left(x_{1}, \cdots, x_{n}\right) \rightarrow A_{s_{1} \cdots s_{m}}\left(z_{1}, \cdots, z_{m}\right),
$$

then

$$
T_{s_{1} \cdots s_{m}}: A\left(f_{1}\left(x_{1}\right), \cdots, f_{n}\left(x_{n}\right)\right) \rightarrow A_{s_{1} \cdots s_{m}}\left(f_{s_{1}}\left(z_{1}\right), \cdots, f_{s_{m}}\left(z_{m}\right)\right) .
$$

This theorem points out a natural method for handling results about the coefficients in a formal power series in noncommuting variables. There are many ways of choosing a set of generating functions for these coefficients. But the method used in the theorem has the distinct advantage of transforming the set of generating functions into a new set in the same way that the related formal power series are transformed into each other. We have restricted ourselves to transformations with a vanishing constant term for reasons of simplicity, and also because we wished to keep the result strictly formal. Transformations with nonzero constant terms would involve us in considerations of infinite sums of the coefficients.

Following the proof of the theorem we give an example of its application.

The proof of the theorem is a simple exercise in manipulating sums. The only sums in which convergence might be a problem are finite.

We assume that $A\left(x_{1}, \cdots, x_{n}\right)$ has the expansion (1). Denote the coefficient of $x^{h}$ in the $i$ th power of $f_{s}(x)$ by $f_{s h}^{(i)}$ :

$$
\left\{f_{s}(x)\right\}^{i}=\sum_{h=i}^{\infty} f_{s h}^{(i)} x^{h} .
$$

Then $A\left(f_{1}\left(x_{1}\right), \cdots, f_{n}\left(x_{n}\right)\right)$ has the expansion

$$
\begin{aligned}
a_{0}+\sum_{m=1}^{\infty} \sum_{\left(s_{1}\right) \in V_{n m}}^{m} \sum_{j=1}^{\infty} \sum_{i_{j}=1}^{\infty} a_{s_{1}} \cdots s_{m}\left(i_{1}, \cdots, i_{m}\right) \\
\cdot \sum_{k=1}^{m} \sum_{h_{k}=i_{k}}^{\infty} f_{s_{1} h_{1}}^{\left(i_{1}\right)} \cdots f_{s_{m} h_{m}}^{\left(i_{m}\right)} x_{s_{1}} x_{1} \cdots x_{s_{m}}^{h_{m}} .
\end{aligned}
$$

Therefore the coefficient of $x_{s_{1}}^{h_{1}} \cdots x_{s_{m}}^{h_{m}}$ in $A\left(f_{1}\left(x_{1}\right), \cdots, f_{n}\left(x_{n}\right)\right)$ is

$$
\sum_{j=1}^{m} \sum_{i_{j}=1}^{h_{j}} a_{s_{1}} \cdots s_{m}\left(i_{1}, \cdots, i_{m}\right) f_{s_{1} h_{1}}^{\left(i_{1}\right)} \cdots f_{s_{m} h_{m}}^{\left(i_{m}\right)}
$$

This coefficient is well defined because the sums have a finite number of terms.

It follows that $T_{s_{1}} \cdots s_{m}$ takes $A\left(f_{1}\left(x_{1}\right), \cdots, f_{n}\left(x_{n}\right)\right)$ into 


$$
\begin{aligned}
\sum_{k=1}^{m} \sum_{h_{k}=1}^{\infty} & \sum_{j=1}^{\infty} \sum_{i_{j}=1}^{h_{j}} a_{s_{1}} \cdots s_{m} \\
& \left.=\sum_{j=1}^{\infty} \sum_{i_{j=1}}^{\infty} a_{s_{1}} \cdots s_{m}\left(i_{1}, \cdots, i_{m}\right) f_{s_{1} h_{1}}^{\left(i_{1}\right)} \cdots f_{s_{m} h_{m}} z_{1}\right)\left\{f_{s_{i}}\left(z_{1}\right)\right\}^{\left.i_{m}\right)} \cdots\left\{f_{s_{m}}\left(z_{m}\right)\right\}^{h_{1}} i_{m}^{h_{m}} .
\end{aligned}
$$

This last expression is just $A_{s_{1}} \cdots s_{m}\left(f_{s_{1}}\left(z_{1}\right), \cdots, f_{s_{m}}\left(z_{m}\right)\right)$, as one can see by comparing it with the right-hand expression in (2). This proves the theorem.

We can now apply this theorem to the formal power series for $\log F(x) G(y)$ with $F(0)=G(0)=1$. Since we have only two variables, $V_{n m}$ has only two elements: $(1,2,1,2, \cdots)$ denoting monomials beginning with a power of $x$, and $(2,1,2,1, \cdots)$ denoting monomials beginning with a power of $y$. In order to avoid this cumbersome notation, which is necessary only when we have many variables, we will use the subscript $x$ instead of $1212 \ldots$ and the subscript $y$ instead of $2121 \cdots$.

Let $b_{x}\left(i_{1}, \cdots, i_{m}\right)$ denote the coefficient of $x^{i_{1}} y^{i_{2}} \cdots$ in $\log F(x) G(y)$, with $b_{y}\left(i_{1}, \cdots, i_{m}\right)$ similarly defined. We want to find an expression for

$$
B_{x}\left(z_{1}, \cdots, z_{m}\right)=\sum_{j=1}^{m} \sum_{i_{j}=1}^{\infty} b_{x}\left(i_{1}, \cdots, i_{m}\right) z_{1}^{i_{1}} \cdots z_{m}^{i_{m}},
$$

and for $B_{y}\left(z_{1}, \cdots, z_{m}\right)$, the generating function of $b_{y}\left(i_{1}, \cdots, i_{m}\right)$.

We shall use the following results from [1]. Let $a_{x}\left(i_{1}, \cdots, i_{m}\right)$ and $a_{y}\left(i_{1}, \cdots, i_{m}\right)$ denote the coefficients in $\log e^{x} e^{y}$, and let $A_{x}\left(z_{1}, \cdots, z_{m}\right)$ and $A_{y}\left(z_{1}, \cdots, z_{m}\right)$ denote their generating functions. Theorem 2 of [1] states that

$$
A_{x}\left(z_{1}, \cdots, z_{m}\right)=\sum_{i=1}^{m} z_{i} e^{m^{\prime} z_{i}} \prod_{j \neq i}\left(e^{z_{i}}-1\right)\left(e^{z_{i}}-e^{z_{j}}\right)^{-1}
$$

and

$$
A_{y}\left(z_{1}, \cdots, z_{m}\right)=\sum_{i=1}^{m} z_{i} e^{m^{\prime \prime} z_{i}} \prod_{j \neq i}\left(e^{z_{i}}-1\right)\left(e^{z_{i}}-e^{z_{j}}\right)^{-1}
$$

where $m^{\prime}=[m / 2]$ and $m^{\prime \prime}=[(m-1) / 2]$, with $[t]$ denoting the greatest integer in $t$.

In order to apply the theorem of this paper we define $f(x)$ and $g(y)$ by

$$
F(x)=e^{f(x)}, \quad G(y)=e^{g(y)}
$$

and note that $f(0)=g(0)=0$. Then we can transform the generating 
functions for the coefficients in $\log e^{x} e^{y}$ into the generating functions for the coefficients in $\log e^{f(x)} e^{g(y)}=\log F(x) G(y)$ directly:

$$
B_{x}\left(z_{1}, \cdots, z_{m}\right)=A_{x}\left(f\left(z_{1}\right), g\left(z_{2}\right), \cdots,[f, g]\left(z_{m}\right)\right)
$$

and

$$
B_{y}\left(z_{1}, \cdots, z_{m}\right)=A_{y}\left(g\left(z_{1}\right), f\left(z_{2}\right), \cdots,[g, f]\left(z_{m}\right)\right)
$$

where $[f, g]\left(z_{m}\right)$ denotes $f\left(z_{m}\right)$ if $m$ is odd and $g\left(z_{m}\right)$ if $m$ is even.

Applying (5) and (6) to (3) we get

$$
\begin{aligned}
B_{x}\left(z_{1}, \cdots,\right. & \left., z_{m}\right) \\
= & \sum_{i=1}^{m-m^{\prime}}\left\{F\left(z_{2 i-1}\right)\right\}^{m^{\prime}} \log F\left(z_{2 i-1}\right) \prod_{j=1 ; j \neq i}^{m-m^{\prime}} \frac{F\left(z_{2 j-1}\right)-1}{F\left(z_{2 i-1}\right)-F\left(z_{2 j-1}\right)} \\
& \cdot \prod_{j=1}^{m^{\prime}} \frac{G\left(z_{2 j}\right)-1}{F\left(z_{2 i-1}\right)-G\left(z_{2 j}\right)}+\sum_{i=1}^{m^{\prime}}\left\{G\left(z_{2 i}\right)\right\}^{m^{\prime}} \log G\left(z_{2 i}\right) \\
& \cdot \prod_{j=1}^{m-m^{\prime}} \frac{F\left(z_{2 j-1}\right)-1}{G\left(z_{2 i}\right)-F\left(z_{2 j-1}\right)} \prod_{j=1 ; j \neq i}^{m^{\prime}} \frac{G\left(z_{2 j}\right)-1}{G\left(z_{2 i}\right)-G\left(z_{2 j}\right)} .
\end{aligned}
$$

Applying (5) and (7) to (4) we see that $B_{y}\left(z_{1}, \cdots, z_{m}\right)$ can be obtained from (8) by interchanging $F$ and $G$ throughout and then replacing the exponent $m^{\prime}$ in the first term of each sum by $m^{\prime \prime}$.

Since $F(0)=1$ the power series for $\log F(z)$ is

$$
\log F(z)=\sum_{k=1}^{\infty} \frac{(-1)^{k-1}}{k}(F(z)-1)^{k} .
$$

Thus, an example of (8) is

$$
\begin{aligned}
B_{x}\left(z_{1}, z_{2}\right)= & F\left(z_{1}\right)\left(G\left(z_{2}\right)-1\right) \sum_{k=1}^{\infty} \frac{(-1)^{k-1}}{k} \cdot \frac{\left(F\left(z_{1}\right)-1\right)^{k}-\left(G\left(z_{2}\right)-1\right)^{k}}{F\left(z_{1}\right)-G\left(z_{2}\right)} \\
& -\log G\left(z_{2}\right),
\end{aligned}
$$

as the reader can verify.

The generating function (8) shows that $b_{x}\left(i_{1}, \cdots, i_{m}\right)$ is left fixed by any permutation of the odd indexed $i_{j}$ and any permutation of the even indexed $i_{j}$. For example, $b_{x}\left(i_{1}, i_{2}, i_{3}, i_{4}, i_{5}\right)=b_{x}\left(i_{5}, i_{4}, i_{1}, i_{2}, i_{3}\right)$. The same is true of $b_{y}\left(i_{1}, \cdots, i_{m}\right)$. If $F(z)=G(z)$ then any permutation of the $i_{j}$ leaves the coefficients unchanged. If $F(z)=G(z)$ and $m$ is odd (so that $m^{\prime}=m^{\prime \prime}$ ) then $b_{x}\left(i_{1}, \cdots, i_{m}\right)=b_{y}\left(i_{1}, \cdots, i_{m}\right)$.

\section{REFERENCE}

1. K. Goldberg, The formal power series for $\log e^{x} e^{y}$, Duke Math. J. vol. 23 (1956) pp. 13-22.

National Bureau of Standards 